\title{
CrystEngComm
}

Check for updates

Cite this: CrystEngComm, 2022, 24, 1989

Received 28th December 2021 Accepted 4th February 2022

DOI: 10.1039/d1ce01721g

rsc.li/crystengcomm

\section{Impurity incorporation in solution crystallization: diagnosis, prevention, and control}

\author{
Gerard Capellades, (iD*a Jacob O. Bonsu ${ }^{a}$ and Allan S. Myerson (iD*b
}

\begin{abstract}
Despite their widespread use for purification, our current methods for the development of solution crystallization processes lack a sufficient understanding on how impurities incorporate in growing crystals. This is, in part, due to the large number of mechanisms often encountered for impurity incorporation, and due to limitations in our methods for diagnosis of those mechanisms. These limitations propagate into largely empirical process development strategies, which are still based on trial and error and centered on solvent selection. This manuscript highlights recent developments in the diagnosis, prevention, and control of impurity incorporation during batch and continuous crystallization. The goal is to provide process development scientists with an updated toolkit for understanding how specific impurities are retained in the solid product, and to review recent prevention and control strategies that may be used to improve crystal purity in industrial crystallization processes.
\end{abstract}

\section{Introduction}

Crystallization as a separation process plays a critical role in the manufacturing of bulk chemicals, food products, fine chemicals, and pharmaceuticals. ${ }^{1,2}$ This unit operation is used for purification, but also as an isolation step for the separation of powders with the desired crystal size, habit, and structure. Currently, many of the products based on fine chemicals, for instance, photographic materials, explosives, and dyes, necessitate crystallization in their fabrication, and approximately $90 \%$ of small-molecule pharmaceuticals include drug substances in the crystalline solid form. ${ }^{1}$ As a result, manufacturers strive to control the crystallization process to attain products with reproducible and desired solid-state properties.

In recent years, there has been a significant improvement in our understanding of solution crystallization processes. This is, in part, driven by an increasing need to design robust separation processes in pharmaceutical manufacturing, and by a push towards continuous manufacturing. ${ }^{3-6}$ Quality attributes like bioavailability and stability of pharmaceutical powders are directly related to the solid-state properties of the crystals, including crystal size, habit, and form. ${ }^{7-9}$ Those attributes can be controlled by adjusting crystallization conditions and the addition of in situ and post-crystallization

\footnotetext{
${ }^{a}$ Department of Chemical Engineering, Henry M. Rowan College of Engineering, Rowan University, Glassboro, New Jersey 08028, USA.

E-mail: capellades@rowan.edu

${ }^{b}$ Department of Chemical Engineering, Massachusetts Institute of Technology, Cambridge, Massachusetts 02139, USA.E-mail: myerson@mit.edu
}

mechanical processing steps. ${ }^{10-14}$ However, in contrast with the literature on crystallization for the control of crystal size, habit, and form, the amount of work relative to crystallization as a purification process is relatively scarce.

From a medical perspective, control of impurities in a manufacturing process is especially critical for products that should be fit for human consumption, and the presence of unacceptable levels of genotoxic impurities has been a reason for recent pharmaceutical recalls. ${ }^{15-17}$ Similarly, from the process perspective, traces of impurities have been shown to play a significant role in modifying the thermodynamics and kinetics of crystallization processes. ${ }^{18-23}$ The identification and control of impurity profiles through manufacturing plants is a critical step to ensure a robust operation and accurate control of the propagation of process disturbances. ${ }^{24,25}$

In the manufacturing of small-molecule pharmaceuticals, even a small number of synthesis steps can quickly lead to the generation of dozens of impurities, often with unknown composition or toxicological information. ${ }^{26-30}$ Consequently, most recent examples of end-to-end synthetic pharmaceutical manufacturing involve a number of crystallization steps where the main role is to purify an intermediate product, limiting the accumulation of impurities along the main process stream. ${ }^{25,26,31-38}$ Despite the widespread use of these purification steps, our understanding of how impurities incorporate in crystals is far behind, current models for predicting purity in solution crystallization are limited to a narrow design space, ${ }^{25,28}$ and process design is still largely based on extensive solvent screenings and trial and error. ${ }^{24,26,38-41}$ This is, in part, due to the number and complexity of mechanisms by which impurities incorporate 
in growing crystals, and the limited understanding of kineticdriven interactions between impurities and solute molecules in the crystal-solution interphase.

This manuscript highlights recent advances in the diagnosis and control of impurity incorporation during solution crystallization of small organic molecules. The theory behind those mechanisms is only briefly covered to the extent that is necessary to understand the rest of this manuscript, but it is described in more detail in recent literature. ${ }^{42,43}$ The second half of the manuscript is centred on the recent publications in the context of impurity incorporation and crystallization, reviewing current efforts and novel strategies for prevention and control.

\section{Impurity incorporation in solution crystallization}

When it comes to impurity incorporation mechanisms, it is important to make a distinction between the manufacturing of single crystals and bulk manufacturing. For processes involving the isolation of a crystalline product, in their broadest sense, impurities can end in the solid product by incorporating inside of individual crystals, by adhering to the surface of those crystals, by entrapment within aggregates, or by forming their solid phase as a second precipitate. A summary of the individual impurity incorporation mechanisms is provided in Fig. 1.

For impurity incorporation outside the crystals, i.e. by agglomeration, co-precipitation, or adhering mother liquor,

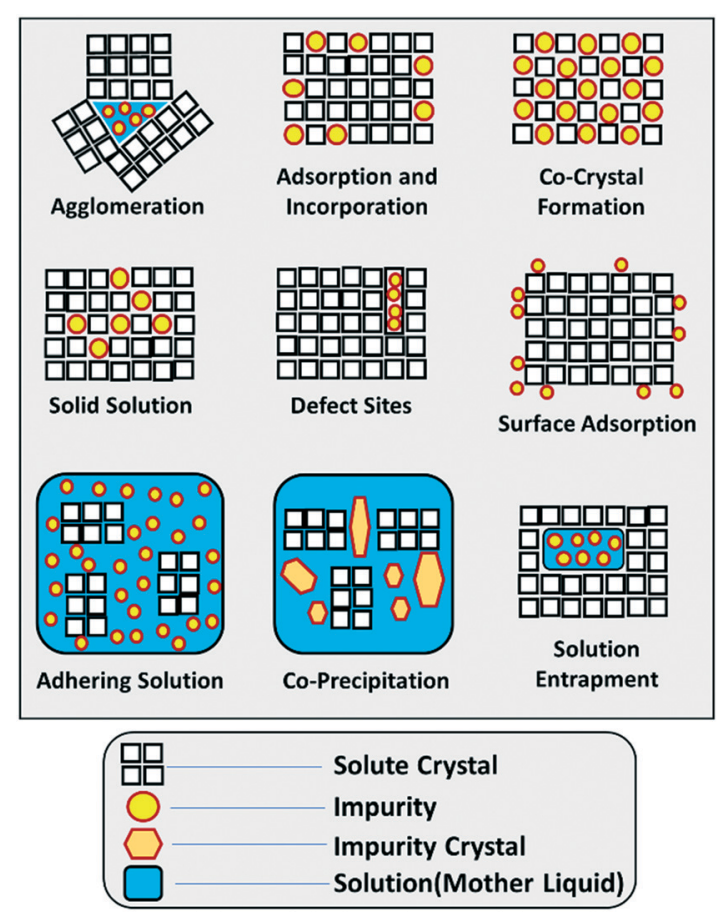

Fig. 1 Schematic illustration of the different impurity incorporation mechanisms in solution crystallization. Inspired by Urwin et al. and expanded for this work. ${ }^{44}$ the mechanisms are fairly straight-forward. The crystallization mother liquor tends to be rich in impurities that have been rejected from the crystalline phase. Thus, the formation of aggregates during crystallization has the possibility of entrapping this mother liquor, decreasing the overall purity of the solid product. Methods for preventing aggregate formation are widely available in the literature and fall out of the scope of this work. ${ }^{44-49}$ Similarly, when the mother liquor is filtered from the solids, adequate washing steps must be in place to wash out the adhering liquid while preventing the precipitation of impurities by anti-solvent effects. ${ }^{20,40}$ In this area, impurity precipitation (either during crystallization, or during washing) is driven by the generation of a supersaturated state for those impurities. If their concentration is too high, or the solubility in the crystallization solvent is too low, impurities may precipitate together with the product of interest, generating a powder that contains multiple solid phases.

A similar impurity incorporation mechanism emanates from solution entrapment, and commonly occurs for systems with porous or large crystals, where a portion of the liquid phase is entrapped in the solid product and is not easily washed away. ${ }^{42,44}$ Inclusions stem from non-equilibrium processes and may be categorized as primary (those formed during crystal growth) or secondary (those formed after crystal growth). One probable account for primary inclusion formation could be due to the disproportionate growth rates at the surface of crystals, mostly with the edges and corners growing quicker than the centre of the faces, leading to cavities formation which are ultimately sealed as growth proceeds. $^{2,43,50}$

The most intricate impurity incorporation mechanisms arguably take place when impurities incorporate inside the crystal. In this area, several mechanisms have been identified in the past, and the distinction between those can be experimentally challenging with our current understanding of those mechanisms. It is important to note that, while the crystalline product is often analysed as a powder on the basis of an average purity, impurities don't necessarily incorporate evenly throughout the crystal lattice. Solute molecules follow a specific order within a crystal lattice. Thus, threedimensional crystals presenting multiple faces will present a unique surface chemistry and a different rate of crystal growth in each face, ultimately defining crystal habit. ${ }^{49,51}$ When it comes to interactions with impurities, some impurities may preferentially interact with certain faces, either because of their growth rate or because of their surface chemistry (e.g. exposed functional groups). Similarly, differences in kinetics throughout crystal growth may lead to different impurity profiles in a grown crystal, generating a core rich in impurities and an increasing purity as we approach the crystal surface. ${ }^{52}$ These mechanisms will lead to a heterogeneous distribution of impurities within the crystal lattice (Fig. 2), with some areas of the crystal presenting impurity concentrations orders of magnitude higher than other areas. ${ }^{53}$ 


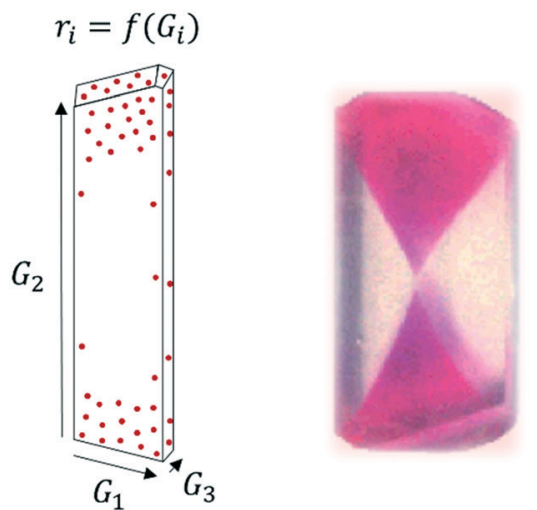

Fig. 2 Left: Face-dependent crystal growth $\left(G_{i}\right)$ and impurity incorporation $\left(r_{i}\right)$ in a growing crystal. Right: Example of facedependent incorporation of sulforhodamine $\mathrm{B}$ in a $\mathrm{K}_{2} \mathrm{SO}_{4}$ crystal ladapted with permission from Kahr and Gurney. ${ }^{54}$ Copyright 2001 American Chemical Society].

A practical analysis of face-dependent impurity incorporation during industrial crystallization development is currently limited by the available diagnosis methods centred on powder analysis. However, it may be a critical step for the improved understanding of impurity incorporation in an inherently heterogeneous system. For more information and examples on face-dependent impurity incorporation, an excellent review on the topic was published by Kahr and Gurney, with focus on the incorporation of dyes in growing crystals. $^{54}$

One of the main mechanisms for impurity incorporation inside a crystal is the formation of solid solutions, where impurities are uniformly distributed within the crystal lattice. These are usually presented as regions with high impurity contents within the crystal. Solid solutions can be substitutional or interstitial. In the first case, the impurity has a similar size and molecular properties as the solute molecule and incorporates by taking the solute's place in the crystal lattice. For interstitial solid solutions, the impurity enters the interstices of the crystal lattice, further disrupting the crystal order. ${ }^{43}$ True solid solutions are energetically unfavourable, and thus they are not expected to occur as a main mechanism in kinetic-driven processes. Small amounts of impurities $(<1 \%)$ are often observed within the crystals, which could be indicative of incorporation in defect sites instead. However, some solid solutions have been observed in systems with structurally similar impurities. ${ }^{53,55-60}$

Similarly, it is important to note that impurities may form co-crystals with the solute system under the appropriate crystallization conditions. These are homogenous crystalline structures comprising a neutral co-former and a neutral target held together by a non-covalent interactive bond. ${ }^{61,62}$ The unique structural orientation of co-crystals is due to the non-covalent interactions between various reacting species that result in the systematic integration of both compounds (target and co-former) into the crystal lattice. ${ }^{62,63}$ For an excellent lattice substitution to take place, the incorporated impurity usually is of similar size and structure to the target compound. ${ }^{61}$ Forming co-crystals of active pharmaceutical ingredients (APIs) with a specially selected co-former is a known approach to enhance crystal quality attributes. However, the target molecule sometimes forms insoluble cocrystals with an impurity. ${ }^{61,64-66}$ For example, Ranganathan et al. observed the formation of a toxic 1:1 co-crystal of melamine and cyanuric acid, which was practically insoluble in the crystallization solvent. ${ }^{67}$

Defects in crystals are readily formed from stresses induced by supersaturation, temperature, and mechanical action, and they can act as a hideout for impurities to incorporate in the crystal. ${ }^{51}$ Edge dislocation is a usual position of impurity uptake in crystals structure essentially because of its unique structure. ${ }^{68}$ The insertion of an extra lattice plane in edge dislocations, compresses the crystal lattice by generating several sites on the surface that can favourably concentrate impurities.

Finally, impurities may adsorb on the crystal surface if they have a sufficiently high binding energy with one or more of the crystal faces. ${ }^{49,51}$ The accumulation of adsorbed impurity molecules at the surface of the growing crystals may precede their incorporation in the crystal lattice as a new layer of solute molecules attaches in the growing crystal face. This mechanism, predominant at slow crystal growth rates, may be hard to differentiate from solid solutions that would also be expected at near-equilibrium. The adsorbed impurities have also been reported to play a role inhibiting crystal growth, and thus they may have an impact on crystallization yield, particle size, and crystal habit. ${ }^{69-71}$

\section{Diagnosis of impurity incorporation mechanisms}

The diagnosis of how impurities incorporate in the growing solute is a critical step for the selection of adequate control strategies that are directed to the root cause of impurity incorporation. For example, a co-precipitating impurity can be easily removed by tuning the crystallization solvent so that this species becomes undersaturated, or by the selection of a proper wash solvent in the subsequent filtration process. ${ }^{38}$ Similarly, impurity incorporation driven by agglomeration would need to be handled via mitigation of this phenomenon. Because industrial solution crystallization mostly deals with the formation of powders instead of single crystals, several methods have been described in the literature for the diagnosis of impurity incorporation mechanisms from powder analysis.

\subsection{Process imaging}

Process imaging methods provide an advantage over the other methods described in this work, as they do not generally require separate off-line experiments for the diagnosis of impurity incorporation and they are not limited to the study of crystal purity. Several tools have been used for 
imaging crystallization processes in situ, including probes and integrated crystallization systems. ${ }^{13,72-76}$ While the focus is generally control of crystal size and habit, process imaging can aid in the detection of impurity incorporation mechanisms by acting as a tool to detect solution inclusions, aggregate formation, or co-precipitation. The latter can be presented as crystals with a different morphology or optical properties, but care should be taken to avoid confusing polymorphs with co-precipitating impurities. A recent example was the detection of co-precipitating phenylglycine impurities during the crystallization of cephalexin monohydrate. For this system, Salami and co-workers used a convolutional neural network (CNN) to identify the formation of phenylglycine crystals from process images taken in situ (Fig. 3). ${ }^{77}$ This detection can be used as a basis for corrective measures like temperature cycling or modifications in solvent composition.

Due to its non-invasive nature, it is recommended to utilize process imaging whenever possible, as it provides valuable information about the crystallization behaviour that is hard to obtain with off-line tests. However, note that this technique is limited to the detection of co-precipitating impurities, solution entrapment, and aggregation, and it may be flawed if co-precipitating impurities present similar optical properties as the crystallizing solute. In those situations, the pairing with Raman spectroscopy could aid the detection of the new solid phase. ${ }^{13,14}$

\subsection{Dry powder analysis}

Similar to the use of process imaging, optical microscopy on off-line samples can aid in the detection of aggregates, ${ }^{25} \mathrm{co}^{-}$ precipitates (Fig. 3), ${ }^{77}$ and solution entrapment. ${ }^{78}$ For the analysis of dry powders by microscopy, it is important to mitigate the formation of aggregates or co-precipitates during washing and drying, which could induce bias in the results. Alternative methods have been used where the suspension is carefully sampled directly from the crystallizer, and diluted in a large amount of saturated solution to simultaneously
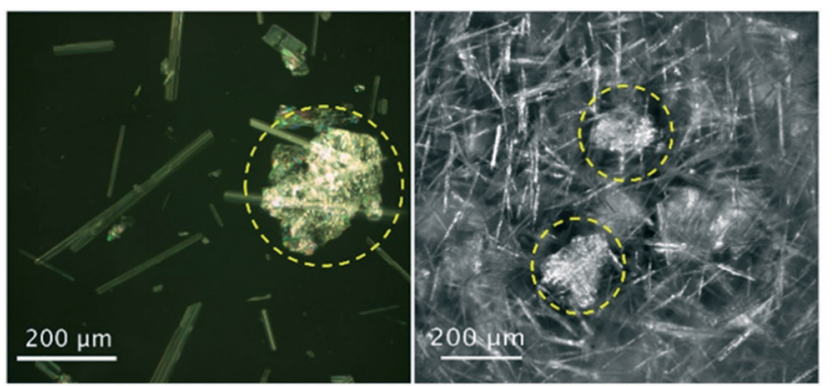

Fig. 3 Optical microscopy (left) and in situ process images (right) of co-precipitates of phenylglycine impurities (circled) during crystallization of needle-like cephalexin monohydrate crystals [adapted with permission from Salami et al. ${ }^{77}$ Copyright 2021 American Chemical Society]. quench crystallization and dilute the solids concentration to a level that facilitates imaging. ${ }^{10}$

Analysis of powder mixtures by X-ray powder diffraction (XRPD) can also reveal information on crystallizing impurities, seen as the appearance of a new pattern revealing a mixture of crystalline phases, ${ }^{79-84}$ or the formation of a cocrystal. ${ }^{85}$ In addition, solid solutions have been shown to affect the diffraction peak positions by expansion or contraction of the lattice, ${ }^{86}$ as well as a reduction in peak intensities due to the loss of crystallinity. ${ }^{53,87} \mathrm{X}$-ray diffraction has also been used in single crystals, for specific faces showing a high degree of impurity incorporation. In those systems, X-ray diffraction can be used to identify the atomic positions of the impurities in the host crystal. ${ }^{43}$

Differential scanning calorimetry (DSC) analysis of crystallized powders can also be used to detect the incorporation of impurities. The lattice incorporation of impurities can have a small effect on the observed melting point and enthalpy of fusion, ${ }^{53,84,87}$ and the co-precipitation of impurities can be seen as peak doubling. ${ }^{88}$ However, due to the possibility of peak doubling by melting and recrystallization of the solute crystals, care should be taken when interpreting DSC data on systems potentially presenting impurity co-precipitation and polymorphism.

\subsection{Powder dissolution}

Off-line tests based on powder dissolution are perhaps the most accessible method for diagnosing the location of impurities within a system. In these tests, the dry crystalline powder containing solute and impurities is slowly dissolved by gradual solvent addition, and the concentration of dissolved impurities in the washed solvent (or, alternatively, the concentration of impurities in the solids) is tracked as a function of the amount of solvent added, using analytical methods like high performance liquid chromatography (HPLC). This analysis can be based on a relative area percent (LCAP) or, if the impurities are known and available, based on their actual concentrations using a separate calibration

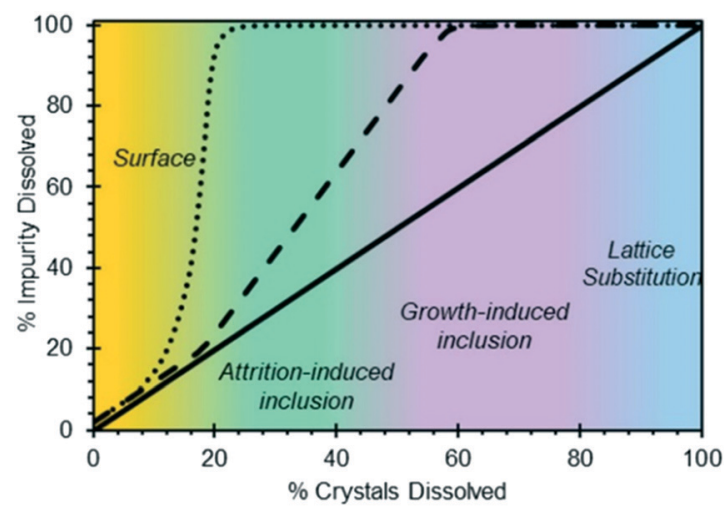

Fig. 4 Hypothesized impurity distribution maps based on progressive powder dissolution tests [reprinted with permission from Urwin et al. ${ }^{44}$ Copyright 2020 American Chemical Society]. 
line. ${ }^{89}$ The results are analysed using a component phase balance between the solids and the liquid phase, providing with a trend in the impurities' dissolution rate relative to the solute's dissolution rate. A high degree of impurity dissolution in the early dissolution steps is generally assumed to indicate that the impurities are located at the surface of the crystal (Fig. 4). Similarly, impurities dissolving towards the later steps would indicate that they are predominantly inside the crystal, in areas that are sometimes not accessible during the early dissolution steps. ${ }^{44,51}$

It is important to note that these methods rely on the assumption that crystal faces dissolve evenly and that the presence of impurities does not affect the dissolution rate of the impure crystal faces. Wang et al. conducted dissolution studies of crystallized salicylic acid, which presented elongated crystals with an impure crystal core. ${ }^{52}$ Upon the addition of a small amount of solvent, the core that was rich in impurities dissolved first, generating macrotubular crystals with a significantly higher average purity (Fig. 5).

It has been widely reported that the presence of impurities in a crystallization system can have a significant impact on solubility and kinetics. ${ }^{18,19,21,90-92}$ Similarly, the incorporation of foreign species in a pure crystal can be disruptive for the crystal lattice and is energetically unfavourable. It is thus not hard to believe that, if impurities selectively incorporate at the core of the crystal or at given crystal faces, ${ }^{54}$ those areas may be the first to dissolve when presented with a solvent or undersaturated solution. Considering that progressive dissolution studies have been used for decades, ${ }^{49,51,93}$ this recent evidence implies that several systems presenting internal impurity gradients could have been misdiagnosed as surface impurities. As a preventive measure for systems exhibiting a similar behaviour, it is recommended to pair powder dissolution studies with optical microscopy, to ensure that crystal habit

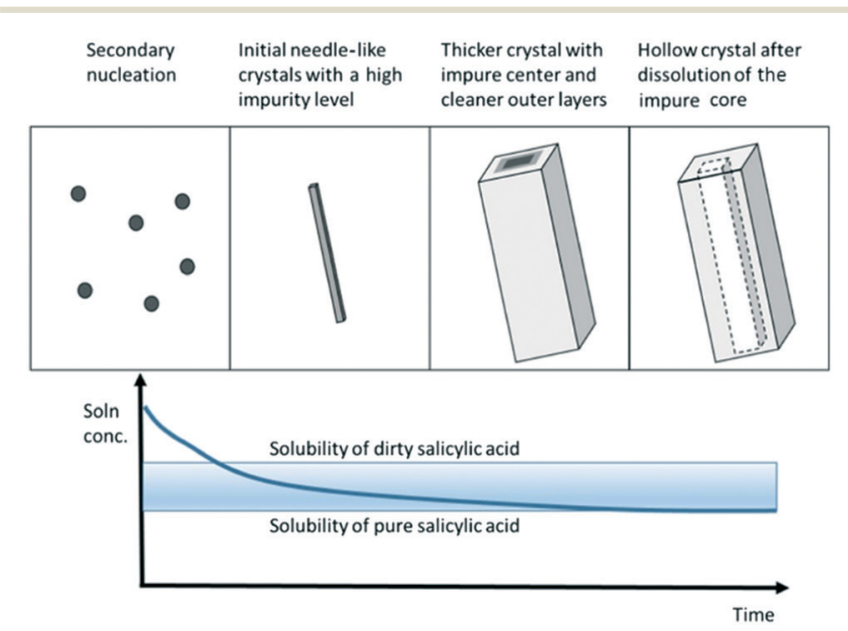

Fig. 5 Schematic illustrating the formation of macrotubular crystals of salicylic acid by crystallization of needle-like crystals and subsequent hollowing during dissolution [reprinted with permission from Wang et al. ${ }^{52}$ Copyright 2021 American Chemical Society]. remains unaltered during the dissolution tests, and to verify the absence of new cavitation.

A novel type of powder dissolution test, named solubilitylimited impurity purge (SLIP) test, was recently developed by Nordstrom et al., for the detection of co-precipitating impurities in powder mixtures. ${ }^{94}$ The method consists on suspending powder samples in various amounts of the crystallization solvent, centrifuging and filtering those suspensions after they have been aged for at least 24 hours. The method tracks the concentration of impurities in the solid and liquid phases using HPLC. When co-precipitating impurities are present, low amounts of solvent will generate a saturated solution of the impurities, and thus their liquid concentration will remain constant regardless of the dilution factor. This will be true until the solvent volume is enough to fully dissolve the impurities, and at this point, their concentration would drop due to dilution. The start of this drop gives the solubility of the impurity. By tracking the purity of the solid phase, it is also possible to evaluate whether the impurities or the solute are preferentially dissolved in the new solvent.

\subsection{Powder-based impurity adsorption tests}

Diagnosis of impurity adsorption mechanisms is difficult in kinetic-driven systems, where crystal growth can lead to the incorporation of adsorbed impurities in the growing layer. Similarly, the need to differentiate surface impurities from those in adhering mother liquor requires the careful design of a washing step that displaces the adhering mother liquor without inducing precipitation or dissolution of the surface impurities.

Powder-based methods based on the use of different particle sizes are frequently used for the diagnosis of surface impurities. The powder sample can be sieved and the different sieve fractions can be analysed for crystal purity. If the impurities are located at the surface, smaller crystals with a higher surface area per unit volume will present a lower purity. ${ }^{43}$ When using this method, it is important to make sure that impurities did not co-precipitate (as a powder with likely a different size distribution) during crystallization. While lower purities in smaller crystals could be indicative of surface incorporation, lower purities for larger crystals could be a consequence of a spread in nucleation times (older crystals present at the start of crystallization, with different impurity incorporation rates at higher supersaturations), solution entrapment, or agglomeration.

An alternative approach for the simultaneous study of adsorption and adsorption-incorporation mechanisms was recently implemented by Teerakapibal et $a .^{53}$ In their studies, they conducted seeded batch crystallizations with the same amount of regular and ground seeds, and investigated the purity of the final product. If adsorption plays a role in impurity incorporation, the product from the smaller seeds will present a lower purity due to the increased surface area. This approach requires a careful implementation to operate 
within a low, narrow range of supersaturations. This is to mitigate nucleation and to minimize bias from the different desupersaturation profiles that would be expected with two different starting surface areas.

\subsection{Single crystal analysis}

Several analytical methods exist for investigating the incorporation of impurities in single crystals. While industrial solution crystallization deals with powders, the analysis of single crystals is still useful to diagnose the location of impurities within a crystal, and it provides the highest level of fundamental understanding of the relationship between surface chemistry, crystal growth, and impurity incorporation.

Electron microscopy and atomic force microscopy can offer information about the crystal surfaces at the nanometer scale, which can be used to detect defects in the crystals and investigate growth mechanisms in each crystal face. ${ }^{95-97}$ In combination with chemical imaging or etching studies, these techniques could reveal impurity incorporation in defect sites, as well as the relationship between the crystal growth mechanism and impurity incorporation. ${ }^{43}$

A common technique for chemical imaging is the use of Raman spectroscopy on single crystals. Industrial crystallization, especially for small molecule pharmaceutical products, often results in the attainment of crystal sizes at the order of $100 \mu \mathrm{m} .{ }^{98-101}$ With a spatial resolution of appr. 1 $\mu \mathrm{m}$, one can use Raman microscopes to scan the surfaces of those crystals and map the impurity gradients across the crystal. $^{52}$ An alternative technique, although bound to poorer spatial resolutions, is the use of infrared (IR) spectroscopy for the same purpose. ${ }^{43}$

\section{Prevention and control}

\subsection{Solvent selection for crystallization and washing}

While it may not be considered a specific strategy for impurity incorporation control, the importance of a good solvent selection for the prevention of impurity incorporation during crystallization cannot be overstated. The crystallization solvent plays a critical role in defining the attainable quality attributes of the product crystals, ${ }^{99,102}$ affecting solubility, kinetics, crystal habit, and the dominant impurity incorporation mechanism. When impurities are present, an adequate solvent selection may prevent coprecipitation of impurities entirely by keeping them undersaturated. Its direct effects on nucleation and growth kinetics will also play an indirect role in impurity incorporation, by offering control over the crystal surface area (adsorption-driven mechanisms) or minimizing crystal growth to prevent solution entrapment. Similarly, solvent plays a role in the tendency of solutes to aggregate, and recent solvent selection strategies have placed a higher emphasis on this factor. ${ }^{75}$

It is thus not surprising that, when the amount of time and representative crude material are limited in process development, most of the focus is placed on the selection of crystallization and wash solvents. A recent example was reported for the last two iterations of the Pharmacy on Demand (PoD) project, where end-to-end processes for several pharmaceuticals had to be developed in parallel with synthesis steps (limited amounts of crude available for downstream) and within a very limited time frame. In the project's "Demonstration" phase, Rogers et al. presented a downstream process development framework largely based on solvent screenings with small amounts of crude material, and applied it to the development of five downstream processes for different pharmaceuticals. ${ }^{26}$ In the later "GMP" phase, Capellades et al. optimized the crystallization and wash solvent compositions for maximizing yield and purity of a recovery process for Ciprofloxacin, increasing crude purity from $78 \%$ to $97 \%$ in the first crystallization-filtrationwashing cycle, and from $97 \%$ to $99 \%$ in the second cycle. ${ }^{38}$ The solvent selection process started similarly to that from the previous phase, ${ }^{26}$ with an early screening for precipitation solvents at the $10 \mathrm{~mL}$ scale. After selecting two promising solvent compositions for crystallization, and two compositions for washing, a factorial design was conducted to guide the final selection as soon as enough crude became available. An optimal solvent combination was chosen and scaled-up in an off-line process (Fig. 6), before being implemented in end-to-end manufacturing. ${ }^{27,38}$ While development was conducted based on a limited understanding of the mechanisms for impurity incorporation, an important part of the purification efficiency came from the selection of crystallization solvents that mitigated the co-precipitation of challenging impurities, and to the selection of wash steps to re-dissolve organic impurities and inorganic precipitates.

In a similar context, Shahid et al. presented methods for the selection of wash solvents, with focus on mitigating the co-precipitation of impurities while maximizing yield. ${ }^{103}$ The methods are suitable for small amounts of material, as they are centred on small centrifugation vials. The study, demonstrated for acetaminophen and two relevant impurities, is focused on anti-solvent effects and aids in mapping the amount and types of solvents that could be used in a series of washing steps to maximize purity and minimize API losses by dissolution.

\subsection{Predictive models}

While population balance models for crystallization have been available for several decades, ${ }^{104}$ the use of predictive modelling for crystallization process development has expanded significantly in recent years. In their simplest form, crystallization models relate nucleation and growth kinetics to process yield and a unidimensional crystal size distribution, assuming negligible crystal breakage, negligible aggregation, and a constant shape factor. These models largely consist on closing a mathematical loop (Fig. 7), where crystallization kinetics and a measure of time define the 
a)

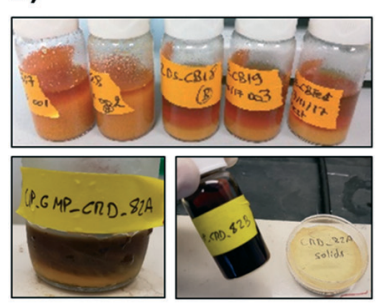

b)

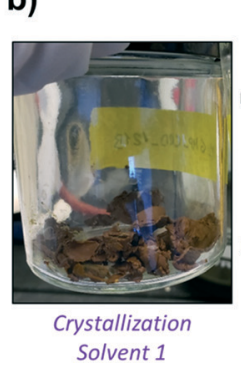

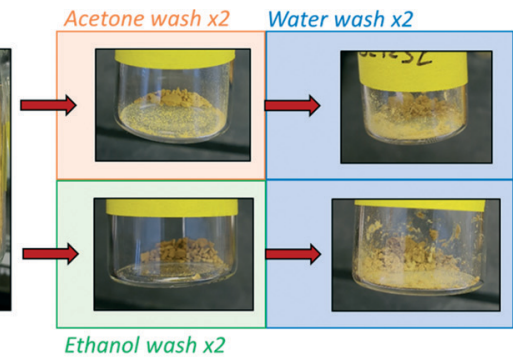

c)

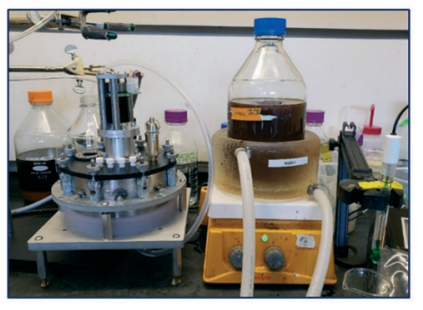

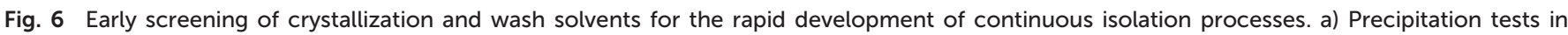

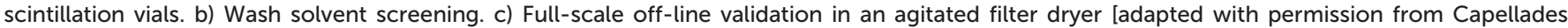
et al. ${ }^{38}$ Copyright 2021 American Chemical Society].

population of crystals in the crystallizer, $n(L)$. With knowledge on the size, shape, and number of crystals at each size range (population), one can calculate suspension properties like the concentration of crystals at a given time $\left(M_{\mathrm{t}}\right)$, as well as their size distribution $(\operatorname{vol}(L))$. Knowing the concentration of crystals and the total solute concentration, $C_{\text {tot }}$, one can calculate how much solute is left in the liquid phase $\left(C_{\mathrm{ml}}\right)$ with a phase balance, and then use $C_{\mathrm{ml}}$ to calculate supersaturation $(\sigma)$ and the change in crystallization kinetics. The population balance equation, $n(L)=f(B, G$, time), depends on the type of crystallizer, but it is generally a function of the investigated crystal size, the rates of nucleation and crystal growth, and a measure of time. ${ }^{51,104}$ Simpler predictive models exist, often based in the method of moments, which sacrifices a prediction of the entire size distribution in exchange for tracking critical metrics like total solids concentration or mean particle size. ${ }^{104-107}$ In addition, variations exist to some of the equations in Fig. 7, like the use of a more accurate phase balance for high solid concentrations, ${ }^{108}$ and other simplifications to the thermodynamic definition of supersaturation that have become a standard practice in crystallization modelling, but have been shown to introduce unnecessary uncertainties in the predicted kinetics. ${ }^{109,110}$
In the context of impurity incorporation, additional equations have been introduced to the crystallization models, often relating predictable metrics like liquid concentrations or crystal growth rates to an average partition coefficient, $\mathrm{DC}_{\mathrm{i}}$, for the impurity (eqn (1)).

$$
\mathrm{DC}_{\mathrm{i}}=\frac{\left(C_{\mathrm{i}} / C_{\text {solute }}\right)_{\text {solid }}}{\left(C_{\mathrm{i}} / C_{\text {solute }}\right)_{\text {liquid }}}
$$

where $C_{\mathrm{i}}$ is the concentration of the impurity and $C_{\text {solute }}$ is the solute concentration.

While impurities do incorporate at different regions within a crystal and within the powder, the modelled $\mathrm{DC}_{\mathrm{i}}$ is an average partition coefficient for the two studied phases: the solids and the liquid. Some mechanistic models do exist and are applied to crystallization from the melt, ${ }^{51}$ however, solution crystallization models for impurity incorporation in recent literature are largely empirical. A popular model was presented by Quon et al. in 2012 for the crystallization of aliskiren hemifumarate. ${ }^{111}$ The model, as shown in eqn (2), was developed from empirical observations of a linear relationship between the impurity concentration in the liquid phase of a crystallizing suspension and the distribution coefficient of that impurity.

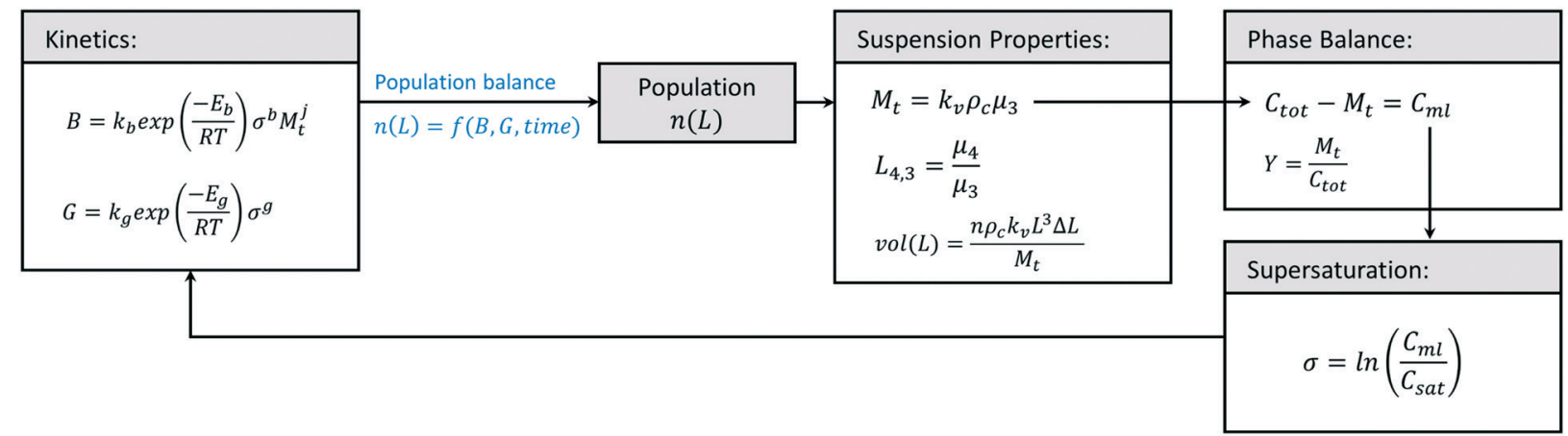

Fig. 7 General shape of solution crystallization models for the prediction of crystallization yield $(Y)$, mean particle size $\left(L_{4,3}\right)$, crystal size distribution $(\operatorname{vol}(L))$, and solids concentration $\left(M_{\mathrm{t}}\right)$ from process conditions like total solute concentration $\left(C_{\text {tot }}\right)$, temperature $(T)$, and crystallization time. The models require knowledge on the system solubility $\left(C_{\text {sat }}\right)$, crystal shape factor $\left(k_{\mathrm{v}}\right)$, crystal density $\left(\rho_{\mathrm{C}}\right)$, the desired distribution channels and their sizes $(L, \Delta L)$, as well as the system's kinetic parameters $\left(K_{\mathrm{b}}, E_{\mathrm{b}}, k_{\mathrm{g}}, E_{\mathrm{g}}, b, g, j\right)$ in order to calculate the evolving nucleation $(B)$ and growth $(G)$ rates, supersaturation $(\sigma)$, mother liquor solute concentration $\left(C_{m l}\right)$, crystal population $(n(L))$, and its population moments $\left(\mu_{\mathrm{i}}=\int n L^{i} d L\right)$. 


$$
\mathrm{DC}_{\mathrm{i}}=a\left(\frac{C_{\mathrm{i}}}{C_{\mathrm{i}}+C_{\text {solute }}}\right)+b
$$

where $a$ and $b$ are constants that can be calibrated from experimental data.

The model presented in eqn (2) can be introduced to the loop in Fig. 7, utilizing $C_{\mathrm{i}}$ and $C_{\text {solute }}$ (as $C_{\mathrm{ml}}$ ) as obtained from the phase balance for the impurity and the solute, respectively. This approach has been recently implemented for the crystallizations of cyclosporine,${ }^{28,112}$ deferasirox, ${ }^{112}$ as well as some process intermediates. ${ }^{25,113}$ In 2017 , Li et al. used this model to predict the purity of cyclosporine purity in a crude system containing 20 known impurities. ${ }^{28}$ They utilized predictive models to optimize the number of crystallization stages and the crystallization conditions, maximizing purity while meeting yield constraints. In 2020, Maloney et al. implemented this model in the prediction of crystal purity for a virtual plant for a carfilzomib intermediate. ${ }^{25}$ They showed that, while the model fits for individual impurities at fixed crystallizer types and scales, a new calibration would be required for each type of crystallizer and even for varying total impurity concentrations in the crude feed (Fig. 8). This is counter-intuitive considering that the model is primarily a function of impurity concentrations, but it is not a surprising behavior for empirical models bound to a narrow design space.

A different empirical model was used in 2019 by Capellades, Wiemeyer, and Myerson, in the crystallization of Ciprofloxacin from crude solutions containing appr. 60 known impurities. ${ }^{18}$ On the observation of crystal growthdriven incorporation for the main impurities, and for the limited amount of experiments that were a consequence of limitations in the amount of crude, the empirical model shown in eqn (3) was implemented for the process.

$$
\mathrm{DC}_{\mathrm{i}}=a G+b
$$

where $G$ was the linear rate of crystal growth in the continuous crystallizer, obtained using a mathematical

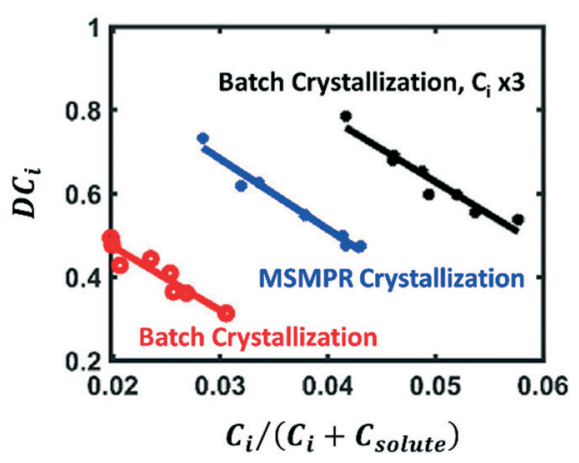

Fig. 8 Fits to eqn (2) for the experimental distribution coefficients of an enone impurity for the nominal concentration level in batch crystallization (red), the nominal concentration level in continuous crystallization (blue), and three times the nominal concentration level in batch crystallization (black) [adapted with permission from Maloney et al. ${ }^{25}$ Copyright 2020 American Chemical Society]. model similar to Fig. 7. The population balance model, including mass balance for solvent and impurity incorporation, was made available as a gPROMS model for that contribution. Beyond this recent literature, other models have been used to model the relationship between impurities and crystal growth. These are described in a recent review, ${ }^{42}$ although most of the available literature is focused on the role of impurities on crystal growth, and not the other way around. Additional mechanistic models have been described elsewhere, ${ }^{43}$ although they often require knowledge on activity coefficients for the solute and impurities, and are generally not used in industrial development.

It is important to note that all the aforementioned empirical models based on an average distribution coefficient, while practical, will be flawed by definition and bound to a narrow design space. Impurities incorporate in growing crystals via a wide variety of mechanisms, and for some of these mechanisms, one crystal face can be responsible for most of the impurity incorporation. ${ }^{54}$ Overall, prediction of impurity incorporation using mechanistic models is a work in progress that requires a better understanding of impurity incorporation mechanisms, an understanding of the role of crystal faces in product purity, as well as highly reliable diagnosis methods to select mechanism-specific models. We have started seeing this modernization in the field of adsorption-driven impurity incorporation, where Borsos, Majumder, and Nagy presented a mechanistic model for the face-dependent impurity incorporation in vitamin B12. This modelling approach implemented a multidimensional population balance and considered both the effects of impurities in crystal growth and the roles of adsorption and growth in crystal purity. ${ }^{114}$

\subsection{Impurity complexation}

A novel method to tackle impurity incorporation during crystallization was initially demonstrated by Hsi et al. for the purification of benzamide and cinnamamide from structurally similar impurities. ${ }^{115}$ Most of the aforementioned mechanisms require impurity molecules to interact with the growing crystal so that they can be incorporated at the surface or lattice. In this context, Hsi et al. hypothesized that co-formers that can form a co-crystal with the impurities will also form a complex in solution, preventing those impurities from incorporating in the growing crystals. This work was a follow-up to prior efforts to isolate impurities by co-crystal formation, as a means to remove them from the crude solution prior to crystallizing the product of interest. ${ }^{116}$ The use of impurity complexation for control of impurity incorporation was further validated for amoxicillin trihydrate crystallization, ${ }^{117}$ showing a greater crystal purity than that obtained after two crystallizations in series without complexing agents.

In 2017, this technology was also applied in continuous crystallization by Vartak and Myerson. ${ }^{118,119}$ To mitigate the common trade-off between crystallization yield and purity, 
a)

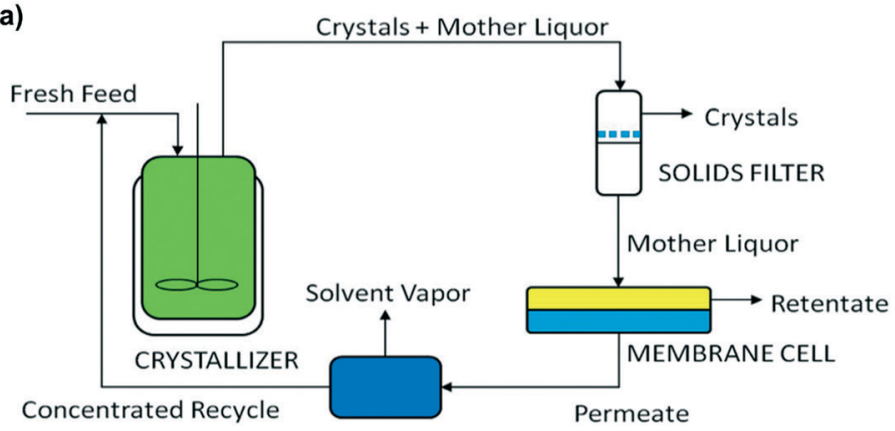

b)

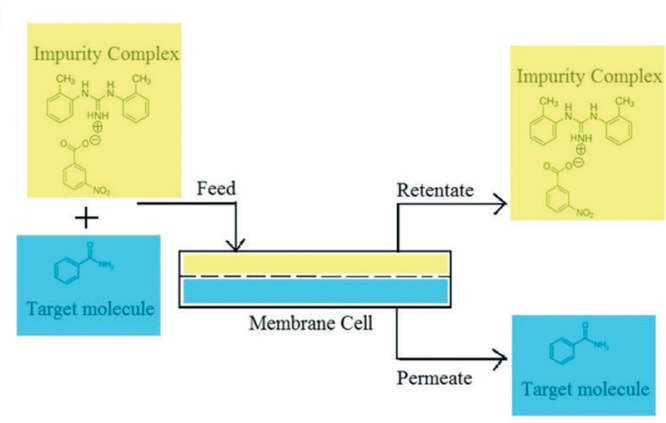

Fig. 9 Prevention of impurity incorporation by complexation in continuous crystallizers with mother liquor recycling. a) Process diagram including continuous crystallization, solids filtration for recovery of solute crystals, nanofiltration for removal of impurity complexes, and permeate concentration prior to mother liquor recycle. b) Close-up of the nanofiltration unit, showing the retention of large impurity complexes and a permeating solution rich in the target solute molecule [adapted with permission from Vartak and Myerson. ${ }^{118,119}$ Copyright 2017 American Chemical Society].

their focus was to allow for solution recycling in systems with impurity complexation. Crystallization of the target molecule, filtration of the pure crystals, and nanofiltration for the removal of impurity complexes were all carried out in a continuous loop (Fig. 9). In this context, the complexing agents play two different roles: first, they prevent impurity incorporation in the crystal lattice by selectively binding with the impurities; then, by isolating the impurities in large complexes, they can be separated from the solvent and solute of interest by membrane filtration, allowing to recycle only the product of interest, and preventing loss of crystal purity due to an increasing impurity concentration in the mother liquor. This recycling approach can also be used without a complexing agent, provided that the impurities have a significantly different molecular size than the solute of interest. ${ }^{120}$

Overall, one of the major advantages from this method is that knowledge of the impurity incorporation mechanism is not required, beyond ensuring that this mechanism is not driven by aggregation, solution entrapment, or adhering mother liquor (where the impurity incorporates as part of the solution and complexes could also be trapped). The main limitation is finding an optimal co-former for impurity complexation, that interacts with the impurities but not with the solute, and that does not incorporate in the crystal lattice. ${ }^{121}$ For known impurity systems, the Cambridge Crystallographic Data Centre (CCDC) can be used to find coformers that would form co-crystals with the impurity, and use those as early candidates for screening. ${ }^{115}$ The measurement of solution interaction energies for potential candidates, and the use of computational models, can facilitate the selection of potential co-formers. ${ }^{121}$ However, for systems with large numbers of unknown impurities, screening for the right co-formers can take a significant amount of time and resources.

\subsection{Slurry aging and temperature cycling}

In those cases where prevention of impurity incorporation is not trivial or practical, an alternative to improve the purity of the final product is to implement strategies to selectively dissolve impurities post-crystallization. Some of these methods have been previously discussed, involving the choice of wash solvents to re-dissolve co-precipitating impurities..$^{38,103}$ A similar method, but based on dissolving solid solutions, was used by Wang et al. for the purification of the salicylic acid crystals shown in Fig. $5 .^{52}$ They suspended the impure crystals in pure water and applied mechanical agitation to promote crystal breakage and expose the impure crystal core to the wash solvent. This allowed for preferential dissolution of the impure core. Slurry aging of impure crystals in a wash solvent has been used for decades, as a means to utilize the dynamic equilibrium to remove an excess of kinetically incorporated impurities and to attain an equilibrium distribution coefficient. ${ }^{122-126}$ However, this method works best when impurities are kinetically incorporated, as the final crystal purity would be driven by thermodynamics.

Temperature cycling can be used as a method to accelerate the purge of impurities in equilibrium suspensions. Instead of relying on Ostwald ripening in the dynamic dissolution-crystallization equilibrium, ${ }^{51}$ the dissolution of impure crystals and re-crystallization at low supersaturations is promoted by heating and cooling cycles, triggering small increases and drops in solubility for both the solute and the impurities. These cycles can also be implemented in supersaturated solutions during crystallization, to tackle several phenomena involved in impurity incorporation. For example, Simone et al. used temperature cycling to minimize agglomeration of piroxicam monohydrate. ${ }^{45}$ In a later contribution, this technique was used to enhance purity during crystallization of vitamin B12 using process analytical technologies (PATs) and feedback control. ${ }^{127}$ The same strategy was demonstrated for improving purity in systems presenting solution entrapment in both aggregates and single crystals. ${ }^{128}$ Presumably, the increase in solubility during the heating cycle aids in dissolving crystalline bridges within aggregates, and partially dissolves some of the formed crystals re-connecting the 
solution pockets with the bulk mother liquor. This was also observed by Kim and co-workers during the crystallization of cyclotrimethylene trinitramine with temperature cycling. ${ }^{129}$ As an alternative, one can use ultrasonication to break crystal aggregates and to expose entrapped solution pockets by crystal breakage. ${ }^{130,131}$

\subsection{Process development workflows}

Despite the wide variety of mechanisms and mechanismdependent approaches to the design of crystallization processes for purification, some workflows do exist as systematic methodologies for the diagnosis and selection of impurity control strategies. Cheng et al. initially presented a workflow for the simultaneous development of crystallization and downstream strategies for control of crystal purity. ${ }^{132}$ The methods are based on an early screening for the understanding of impurity incorporation mechanisms, involving a solubility screening and development of phase diagrams to mitigate impurity co-crystal formation and coprecipitation. This is followed by an early precipitation screening using different solvents and crystallization conditions, and finalized with the optimization of wash solvents and deliquoring conditions.

In 2020, Rogers et al. showed their crystallization development approach as part of the efforts for designing five end-to-end continuous manufacturing processes with strict time and material constraints. ${ }^{26}$ The work includes a downstream development workflow that suggests early development using purified material, and a gradual increase in the use of crude as the synthesis steps are optimized. The workflow is mostly based on solvent selection, first for crystallization and then for the washing steps.

In the same year, Urwin et al. presented a novel development workflow for diagnosis and control of impurity incorporation. ${ }^{44}$ Following the collection of preliminary knowledge, the workflow suggests conducting a set of crystallization experiments that will set the basis for development decisions. The isolated crystals are first examined for agglomerates, suggesting an anti-agglomeration strategy if this is the main mechanism for impurity retention. Then, a set of powder dissolution studies (Fig. 4) are suggested to determine if impurities are adsorbed at the surface, and to map the impurity distribution profile throughout the crystals. This can also be used to detect solvent entrapment. Finally, the workflow describes the identification of solid solutions utilizing binary phase diagrams.

In 2021, Ottoboni et al. published a different development workflow, primarily focused on solvent selection, but utilizing computational tools for the selection and ranking of crystallization and wash solvents. ${ }^{133}$ The focus is to maximize crystallization yield and purity while minimizing the overall solvent consumption. Of the aforementioned workflows, this arguably requires the smallest amount of representative crude. However, this comes at the expense of studying impurity incorporation mechanisms and the possibility of applying mechanism-specific control strategies.

\section{Conclusions}

Diagnosis of impurity incorporation mechanisms during solution crystallization is critical for the optimization of crystallization processes targeting high crystal purities. This is especially important in pharmaceutical processes, where products are subject to stringent quality standards, and where small amounts of certain impurities can have severe detrimental effects on the human body. Literature focused on the understanding and control of impurity incorporation is generally scarce in comparison with that focused on crystal size, habit, or polymorphism. However, in previous years, we have seen an increase in the number of crystallization papers focused on purification, development workflows, and novel approaches for the prevention of impurity incorporation. Despite these advances, there is still a gap in our understanding of impurity incorporation mechanisms, especially in modelling and predicting this impurity incorporation, and in the role of individual crystal faces on product purity. Because of this limited understanding, some of our powder-based methods for impurity detection may misdiagnose the dominant mechanism. This brings additional challenges when our prevention strategies are highly systemdependent, and when extensive screenings are not feasible within the typical time and material constraints in industrial development. This manuscript highlighted these recent efforts, in an attempt to connect new and existing process development scientists with the evolving state-of-the-art, and to facilitate the development of novel work that addresses the existing challenges in diagnosis, modelling, and prevention.

\section{Conflicts of interest}

There are no conflicts to declare.

\section{Notes and references}

1 D. Erdemir, A. Y. Lee and A. S. Myerson, Acc. Chem. Res., 2009, 42, 621-629.

2 H. Lorenz and W. Beckmann, Acta Crystallogr., Sect. C: Cryst. Struct. Commun., 2013, 129-148.

3 K. Plumb, Chem. Eng. Res. Des., 2005, 83, 730-738.

4 D. Zhang, S. Xu, S. Du, J. Wang and J. Gong, Engineering, 2017, 3, 354-364.

5 Y. Ma, S. Wu, E. G. J. Macaringue, T. Zhang, J. Gong and J. Wang, Org. Process Res. Dev., 2020, 24, 1785-1801.

6 C. L. Burcham, A. J. Florence and M. D. Johnson, Annu. Rev. Chem. Biomol. Eng., 2018, 9, 253-281.

7 M. Fujiwara, Z. K. Nagy, J. W. Chew and R. D. Braatz, J. Process Control, 2005, 15(5), 493-504.

8 A. J. Alvarez and A. S. Myerson, Cryst. Growth Des., 2010, 10, 2219-2228.

9 J. Chen, B. Sarma, J. M. B. Evans and A. S. Myerson, Cryst. Growth Des., 2011, 11, 887-895. 
10 G. Capellades, P. U. Joshi, K. Dam-Johansen, M. J. Mealy, T. V. Christensen and S. Kiil, Cryst. Growth Des., 2018, 18, 6455-6469.

11 Y. Yang, L. Song, Y. Zhang and Z. K. Nagy, Ind. Eng. Chem. Res., 2016, 55, 4987-4996.

12 T. C. Lai, S. Ferguson, L. Palmer, B. L. Trout and A. S. Myerson, Org. Process Res. Dev., 2014, 18, 1382-1390.

13 L. Nicoud, F. Licordari and A. S. Myerson, Org. Process Res. Dev., 2019, 23, 794-806.

14 L. Nicoud, F. Licordari and A. S. Myerson, CrystEngComm, 2019, 21, 2105-2118.

15 S. Görög, TrAC, Trends Anal. Chem., 2006, 25, 755-757.

16 Statement alerting patients and health care professionals of NDMA found in samples of ranitidine | FDA.

17 Laboratory Tests | Ranitidine | FDA.

18 G. Capellades, H. Wiemeyer and A. S. Myerson, Cryst. Growth Des., 2019, 19, 4008-4018.

19 C. A. Pons Siepermann and A. S. Myerson, Cryst. Growth Des., 2018, 18, 3584-3595.

20 C. A. Pons Siepermann, S. Huang and A. S. Myerson, Cryst. Growth Des., 2017, 17, 2646-2653.

21 N. Kubota, Cryst. Res. Technol., 2001, 36, 749-769.

22 Z. Berkovitch-Yellin, L. Addadi, M. Idelson, M. Lahav and L. Leiserowitz, Angew. Chem., Int. Ed. Engl., 1982, 21, 1336-1345.

23 K. Sangwal and E. Mielniczek-Brzóska, Cryst. Res. Technol., 2003, 38, 113-124.

24 H. A. Moynihan and D. E. Horgan, Org. Process Res. Dev., 2017, 21, 689-704.

25 A. J. Maloney, E. Içten, G. Capellades, M. G. Beaver, X. Zhu, L. R. Graham, D. B. Brown, D. J. Griffin, R. Sangodkar, A. Allian, S. Huggins, R. Hart, P. Rolandi, S. D. Walker and R. D. Braatz, Org. Process Res. Dev., 2020, 24, 1891-1908.

26 L. Rogers, N. Briggs, R. Achermann, A. Adamo, M. Azad, D. Brancazio, G. Capellades, G. Hammersmith, T. Hart, J. Imbrogno, L. P. Kelly, G. Liang, C. Neurohr, K. Rapp, M. G. Russell, C. Salz, D. A. Thomas, L. Weimann, T. F. Jamison, A. S. Myerson and K. F. Jensen, Org. Process Res. Dev., 2020, 24, 2183-2196.

27 C. Armstrong, Y. Miyai, A. Formosa, D. Thomas, E. Chen, T. Hart, V. Schultz, B. K. Desai, A. Y. Cai, A. Almasy, K. Jensen, L. Rogers and T. Roper, Org. Process Res. Dev., 2021, 25, 1524-1533.

28 J. Li, T. C. Lai, B. L. Trout and A. S. Myerson, Cryst. Growth Des., 2017, 17, 1000-1007.

29 K. P. Cole, J. M. Groh, M. D. Johnson, C. L. Burcham, B. M. Campbell, W. D. Diseroad, M. R. Heller, J. R. Howell, N. J. Kallman, T. M. Koenig, S. A. May, R. D. Miller, D. Mitchell, D. P. Myers, S. S. Myers, J. L. Phillips, C. S. Polster, T. D. White, J. Cashman, D. Hurley, R. Moylan, P. Sheehan, R. D. Spencer, K. Desmond, P. Desmond and O. Gowran, Science, 2017, 356, 1144-1150.

30 S. Mascia, P. L. Heider, H. Zhang, R. Lakerveld, B. Benyahia, P. I. Barton, R. D. Braatz, C. L. Cooney, J. M. B. Evans, T. F. Jamison, K. F. Jensen, A. S. Myerson and B. L. Trout, Angew. Chem., Int. Ed., 2013, 52, 12359-12363.
31 A. Adamo, R. L. Beingessner, M. Behnam, J. Chen, T. F. Jamison, K. F. Jensen, J. M. Monbaliu, A. S. Myerson, E. M. Revalor, D. R. Snead and T. Stelzer, Science, 2016, 352, 61-67.

32 P. Zhang, N. Weeranoppanant, D. A. Thomas, K. Tahara, T. Stelzer, M. G. Russell, M. O'Mahony, A. S. Myerson, H. Lin, L. P. Kelly, K. F. Jensen, T. F. Jamison, C. Dai, Y. Cui, N. Briggs, R. L. Beingessner and A. Adamo, Chem. - Eur. J., 2018, 24, 2776-2784.

33 E. Içten, A. J. Maloney, M. G. Beaver, D. E. Shen, X. Zhu, L. R. Graham, J. A. Robinson, S. Huggins, A. Allian, R. Hart, S. D. Walker, P. Rolandi and R. D. Braatz, Org. Process Res. Dev., 2020, 24, 1861-1875.

34 E. Içten, A. J. Maloney, M. G. Beaver, X. Zhu, D. E. Shen, J. A. Robinson, A. T. Parsons, A. Allian, S. Huggins, R. Hart, P. Rolandi, S. D. Walker and R. D. Braatz, Org. Process Res. Dev., 2020, 24, 1876-1890.

35 C. Hu, C. J. Testa, W. Wu, K. Shvedova, D. E. Shen, R. Sayin, B. S. Halkude, F. Casati, P. Hermant, A. Ramnath, S. C. Born, B. Takizawa, T. F. O'Connor, X. Yang, S. Ramanujam and S. Mascia, Chem. Commun., 2020, 56, 1026-1029.

36 K. P. Cole, B. J. Reizman, M. Hess, J. M. Groh, M. E. Laurila, R. F. Cope, B. M. Campbell, M. B. Forst, J. L. Burt, T. D. Maloney, M. D. Johnson, D. Mitchell, C. S. Polster, A. W. Mitra, M. Boukerche, E. W. Conder, T. M. Braden, R. D. Miller, M. R. Heller, J. L. Phillips and J. R. Howell, Org. Process Res. Dev., 2019, 23, 858-869.

37 B. J. Reizman, K. P. Cole, M. Hess, J. L. Burt, T. D. Maloney, M. D. Johnson, M. E. Laurila, R. F. Cope, C. V. Luciani, J. Y. Buser, B. M. Campbell, M. B. Forst, D. Mitchell, T. M. Braden, C. K. Lippelt, M. Boukerche, D. R. Starkey, R. D. Miller, J. Chen, B. Sun, M. Kwok, X. Zhang, S. Tadayon and P. Huang, Org. Process Res. Dev., 2019, 23, 870-881.

38 G. Capellades, C. Neurohr, N. Briggs, K. Rapp, G. Hammersmith, D. Brancazio, B. Derksen and A. S. Myerson, Org. Process Res. Dev., 2021, 25, 1534-1546.

39 S. Ottoboni, C. J. Price, C. Steven, E. Meehan, A. Barton, P. Firth, A. Mitchell and F. Tahir, J. Pharm. Sci., 2019, 108, 372-381.

40 N. Estime, S. Teychené, J. M. Autret and B. Biscans, Powder Technol., 2011, 208, 337-342.

41 M. Müller, U. Meier, D. Wieckhusen, R. Beck, S. PfefferHennig and R. Schneeberger, Cryst. Growth Des., 2006, 6, 946-954.

42 C. Darmali, S. Mansouri, N. Yazdanpanah and M. W. Woo, Ind. Eng. Chem. Res., 2019, 58, 1463-1479.

43 A. S. Myerson, D. Edemir and A. Y. Lee, Handbook of Industrial Crystallization, Cambridge University Press, Third Edit., 2019.

44 S. J. Urwin, G. Levilain, I. Marziano, J. M. Merritt, I. Houson and J. H. Ter Horst, Org. Process Res. Dev., 2020, 24, 1443-1456.

45 E. Simone, R. Othman, G. T. Vladisavljević and Z. K. Nagy, Pharmaceutics, 2018, 10(1), 17. 
46 E. M. Ålander, M. S. Uusi-Penttilä and Å. C. Rasmuson, Ind. Eng. Chem. Res., 2004, 43, 629-637.

47 R. David, P. Marchal, J. P. Klein and J. Villermaux, Chem. Eng. Sci., 1991, 46, 205-213.

48 R. David, P. Marchal and B. Marcant, Chem. Eng. Technol, 1995, 18, 302-309.

49 J. W. Mullin, Crystallization, Butterworth-Heinemann, Fourth., 2001.

50 K. G. Denbigh and E. T. White, Nature, 1963, 199, 799-800.

51 A. S. Myerson, Handbook of Industrial Crystallization, Butterworth-Heinemann, 2nd edn, 2002.

52 Y. Wang, H. Li, M. Raikes, B. Linehan, J. Robson and F. L. Nordstrom, Cryst. Growth Des., 2021, 21, 4100-4110.

53 R. Teerakapibal, H. Li, B. Linehan and F. L. Nordstrom, Cryst. Growth Des., 2020, 20, 1716-1728.

54 B. Kahr and R. W. Gurney, Chem. Rev., 2001, 101, 893-951.

55 A. J. Florence, C. K. Leech, N. Shankland, K. Shankland and A. Johnston, CrystEngComm, 2006, 8, 746-747.

56 A. J. Cruz Cabeza, G. M. Day, W. D. S. Motherwell and W. Jones, Chem. Commun., 2007, 1600-1602.

57 F. P. A. Fabbiani, L. T. Byrne, J. J. McKinnon and M. A. Spackman, CrystEngComm, 2007, 9, 728-731.

58 D. H. Case, V. K. Srirambhatla, R. Guo, R. E. Watson, L. S. Price, H. Polyzois, J. K. Cockcroft, A. J. Florence, D. A. Tocher and S. L. Price, Cryst. Growth Des., 2018, 18, 5322-5331.

59 A. Burel, N. Couvrat, S. Tisse, Y. Cartigny, P. Cardinael and G. Coquerel, Eur. Phys. J.: Spec. Top., 2017, 226, 869-880.

60 D. E. Horgan, L. M. Crowley, S. P. Stokes, S. E. Lawrence and H. A. Moynihan, in Advanced Topics in Crystallization, ed. Y. Mastai, InTechOpen, 2015.

61 N. Schultheiss and A. Newman, Cryst. Growth Des., 2009, 9, 2950-2967.

62 C. B. Aakeröy and D. J. Salmon, CrystEngComm, 2005, 7, 439-448.

63 M. C. Etter, J. Phys. Chem., 1991, 95, 4601-4610.

64 N. K. Duggirala, M. L. Perry, Ö. Almarsson and M. J. Zaworotko, Chem. Commun., 2015, 52, 640-655.

65 M. Karimi-Jafari, L. Padrela, G. M. Walker and D. M. Croker, Cryst. Growth Des., 2018, 18, 6370-6387.

66 A. Karagianni, M. Malamatari and K. Kachrimanis, Pharmaceutics, 2018, 10(1), 18.

67 A. Ranganathan, V. R. Pedireddi and C. N. R. Rao, J. Am. Chem. Soc., 1999, 121, 1752-1753.

68 J. D. Wright, Molecular crystals, Cambridge University Press, 2nd edn, 1995.

69 R. J. Davey, J. Cryst. Growth, 1976, 34, 109-119.

70 S. Dobberschütz, M. R. Nielsen, K. K. Sand, R. Civioc, N. Bovet, S. L. S. Stipp and M. P. Andersson, Nat. Commun., 2018, 9, 1-6.

71 D. D. Patel and B. D. Anderson, J. Pharm. Sci., 2015, 104, 2923-2933.

72 M. Jiang, X. Zhu, M. C. Molaro, M. L. Rasche, H. Zhang, K. Chadwick, D. M. Raimondo, K. K. Kim, L. Zhou, Z. Zhu, M. H. Wong, D. O'Grady, D. Hebrault, J. Tedesco and R. D. Braatz, Ind. Eng. Chem. Res., 2014, 53, 5325-5336.
73 Y. H. Luo, G. G. Wu and B. W. Sun, J. Chem. Eng. Data, 2013, 58, 588-597.

74 J. Schöll, D. Bonalumi, L. Vicum, M. Mazzotti and M. Müller, Cryst. Growth Des., 2006, 6, 881-891.

75 C. J. Brown, T. Mcglone, S. Yerdelen, V. Srirambhatla, F. Mabbott, R. Gurung, M. L. Briuglia, B. Ahmed, H. Polyzois, J. Mcginty, F. Perciballi, D. Fysikopoulos, P. MacFhionnghaile, H. Siddique, V. Raval, T. S. Harrington, A. D. Vassileiou, M. Robertson, E. Prasad, A. Johnston, B. Johnston, A. Nordon, J. S. Srai, G. Halbert, J. H. ter Horst, C. J. Price, C. D. Rielly, J. Sefcik and A. J. Florence, Mol. Syst. Des. Eng., 2018, 3, 518-549.

76 B. O'Sullivan, P. Barrett, G. Hsiao, A. Carr and B. Glennon, Org. Process Res. Dev., 2003, 7, 977-982.

77 H. Salami, M. A. Mcdonald, A. S. Bommarius, R. W. Rousseau and M. A. Grover, Org. Process Res. Dev., 2021, 25, 1670-1679.

78 A. Waldschmidt, V. Dupray, B. Berton, N. Couvrat, S. Petit and G. Coquerel, J. Cryst. Growth, 2012, 342, 72-79.

79 G. Shete, V. Puri, L. Kumar and A. K. Bansal, AAPS PharmSciTech, 2010, 11, 598-609.

80 M. Mirmehrabi, S. Rohani, K. S. K. Murthy and B. Radatus, Cryst. Growth Des., 2006, 6, 141-149.

81 M. Sun, X. Hu, X. Zhou and J. Gu, Powder Diffr., 2017, 32, 78-85.

82 M. Tiwari, G. Chawla and A. K. Bansal, J. Pharm. Biomed. Anal., 2007, 43, 865-872.

83 M. Sundaram, S. Natarajan, A. G. Dikundwar and H. Bhutani, Powder Diffr., 2020, 35, 226-232.

84 W. Beckmann and H. Lorenz, Chem. Eng. Technol., 2006, 29, 226-232.

85 S. Ishihara, Y. Hattori and M. Otsuka, Spectrochim. Acta, Part A, 2019, 221, 117142.

86 G. Descamps, Y. Cartigny, M. Sanselme, M. N. Petit, S. Petit, E. Aubin and G. Coquerel, Cryst. Growth Des., 2009, 9, 3910-3917.

87 S. P. Duddu and D. J. W. Grant, Thermochim. Acta, 1995, 248, 131-145.

88 M. S. Rabello and J. R. White, Polymer, 1997, 38, 6389-6399.

89 D. D. Caspi and F. L. Nordstrom, Org. Process Res. Dev., 2018, 22, 856-861.

90 A. B. Herhold, D. Ertaş, A. J. Levine and H. E. King, Phys. Rev. E: Stat. Phys., Plasmas, Fluids, Relat. Interdiscip. Top., 1999, 59, 6946-6955.

91 K. Sangwal, K. Wójcik and J. Borc, Cryst. Res. Technol., 2007, 42, 1243-1251.

92 K. Sangwal, Prog. Cryst. Growth Charact. Mater., 1996, 32, $3-43$.

93 L. Addadi, S. Weinstein, E. Gati, I. Weissbuch and M. Lahav, J. Am. Chem. Soc., 1982, 104, 4610-4617.

94 F. L. Nordstrom, B. Linehan, R. Teerakapibal and H. Li, Cryst. Growth Des., 2019, 19(2), 1336-1346.

95 A. McPherson, A. J. Malkin and Y. G. Kuznetsov, Annu. Rev. Biophys. Biomol. Struct., 2000, 29, 361-410.

96 P. Pantaraks and A. E. Flood, Cryst. Growth Des., 2005, 5, 365-371. 
97 A. G. Shtukenberg, L. N. Poloni, Z. Zhu, Z. An, M. Bhandari, P. Song, A. L. Rohl, B. Kahr and M. D. Ward, Cryst. Growth Des., 2015, 15, 921-934.

98 G. Capellades, A. Duso, K. Dam-Johansen, M. J. Mealy, T. V. Christensen and S. Kiil, Org. Process Res. Dev., 2019, 23, 252-262.

99 J. M. Schall, J. S. Mandur, R. D. Braatz and A. S. Myerson, Cryst. Growth Des., 2018, 18, 1560-1570.

100 G. Morris, G. Power, S. Ferguson, M. Barrett, G. Hou and B. Glennon, Org. Process Res. Dev., 2015, 19, 1891-1902.

101 T. Vetter, C. L. Burcham and M. F. Doherty, Chem. Eng. Sci., 2014, 106, 167-180.

102 J. M. Schall, G. Capellades, J. S. Mandur, R. D. Braatz and A. S. Myerson, Org. Process Res. Dev., 2019, 23, 1960-1969.

103 M. Shahid, G. Sanxaridou, S. Ottoboni, L. Lue and C. Price, Org. Process Res. Dev., 2021, 25, 969-981.

104 A. D. Randolph and M. A. Larson, Theory of particulate processes - Analysis and Techniques of Continuous Crystallization, Academic Press, Inc., 1971.

105 N. Bajcinca, S. Qamar, D. Flockerzi and K. Sundmacher, Chem. Eng. Sci., 2011, 66, 3711-3720.

106 C. T. Óciardhá, K. W. Hutton, N. A. Mitchell and P. J. Frawley, Cryst. Growth Des., 2012, 12, 5247-5261.

107 P. J. Frawley, N. A. Mitchell, C. T. Ó'Ciardhá and K. W. Hutton, Chem. Eng. Sci., 2012, 75, 183-197.

108 J. Li, B. L. Trout and A. S. Myerson, Org. Process Res. Dev., 2016, 20, 510-516.

109 J. M. Schall, G. Capellades and A. S. Myerson, CrystEngComm, 2019, 21, 5811-5817.

110 G. Lunardon Quilló, S. Bhonsale, B. Gielen, J. F. Van Impe, A. Collas and C. Xiouras, Cryst. Growth Des., 2021, 21, 5403-5420.

111 J. L. Quon, H. Zhang, A. Alvarez, J. Evans, A. S. Myerson and B. L. Trout, Cryst. Growth Des., 2012, 12, 3036-3044.

112 S. Y. Wong, A. P. Tatusko, B. L. Trout and A. S. Myerson, Cryst. Growth Des., 2012, 12, 5701-5707.

113 H. Zhang, J. Quon, A. J. Alvarez, J. Evans, A. S. Myerson and B. Trout, Org. Process Res. Dev., 2012, 16, 915-924.

114 A. Borsos, A. Majumder and Z. K. Nagy, Cryst. Growth Des., 2016, 16, 555-568.
115 K. H. Hsi, M. Kenny, A. Simi and A. S. Myerson, Cryst. Growth Des., 2013, 13, 1577-1582.

116 K. H. Y. Hsi, K. Chadwick, A. Fried, M. Kenny and A. S. Myerson, CrystEngComm, 2012, 14, 2386-2388.

117 K. H. Y. Hsi, A. J. Concepcion, M. Kenny, A. A. Magzoub and A. S. Myerson, CrystEngComm, 2013, 15, 6776-6781.

118 S. Vartak and A. S. Myerson, Org. Process Res. Dev., 2017, 21, 253-261.

119 S. Vartak and A. S. Myerson, Cryst. Growth Des., 2017, 17, 5506-5516.

120 S. Ferguson, F. Ortner, J. Quon, L. Peeva, A. Livingston, B. L. Trout and A. S. Myerson, Cryst. Growth Des., 2014, 14, 617-627.

121 C. C. Weber, G. P. F. Wood, A. J. Kunov-Kruse, D. E. Nmagu, B. L. Trout and A. S. Myerson, Cryst. Growth Des., 2014, 14, 3649-3657.

122 I. M. Kolthoff and G. E. Noponen, J. Am. Chem. Soc., 1938, 60, 197-201.

123 S. Gaines and A. S. Myerson, AIChE Symp. Ser., 1982, 215, 42-45.

124 P. M. Brown and A. S. Myerson, AIChE J., 1989, 35, 1749-1752.

125 M. Saska and A. S. Myerson, AIChE J., 1987, 33, 848-852.

126 P. M. Brown, M. Marquering and A. S. Myerson, Ind. Eng. Chem. Res., 1990, 29, 2089-2093.

127 E. Simone, W. Zhang and Z. K. Nagy, Cryst. Growth Des., 2015, 15, 2908-2919.

128 A. N. Saleemi, G. Steele, N. I. Pedge, A. Freeman and Z. K. Nagy, Int. J. Pharm., 2012, 430, 56-64.

129 J. W. Kim, J. K. Kim, H. S. Kim and K. K. Koo, Org. Process Res. Dev., 2011, 15, 602-609.

130 T. T. H. Nguyen, A. Khan, L. M. Bruce, C. Forbes, R. L. O'Leary and C. J. Price, Crystals, 2017, 7(10), 294.

131 C. Forbes, T. T. H. Nguyen, R. L. O'Leary and C. J. Price, Proc. Meet. Acoust., 2018, 32, 045017.

132 Y. S. Cheng, K. W. Lam, K. M. Ng and C. Wibowo, AIChE J., 2010, 56, 633-649.

133 S. Ottoboni, B. Wareham, A. Vassileiou, M. Robertson, C. J. Brown, B. Johnston and C. J. Price, Org. Process Res. Dev., 2021, 25, 1143-1159. 